Mouzon, Benoit, Chaytow, Helena, Crynen, Gogce, Bachmeier, Corbin, Stewart, Janice, Mullan, Michael, Stewart, William, and Crawford, Fiona (2013) Repetitive mild traumatic brain injury in a mouse model produces learning and memory deficits accompanied by histological changes. Journal of Neurotrauma, 29 (18). pp. 2761-2773. ISSN 0897-7151

Copyright (c) 2012 Mary Ann Liebert, Inc.

A copy can be downloaded for personal non-commercial research or study, without prior permission or charge

Content must not be changed in any way or reproduced in any format or medium without the formal permission of the copyright holder(s)

When referring to this work, full bibliographic details must be given

http://eprints.gla.ac.uk/72680/

Deposited on: 14 July 2014

Enlighten - Research publications by members of the University of Glasgow http://eprints.gla.ac.uk 


\title{
Repetitive Mild Traumatic Brain Injury in a Mouse Model Produces Learning and Memory Deficits Accompanied by Histological Changes
}

\author{
Benoit Mouzon, ${ }^{1-3}$ Helena Chaytow, ${ }^{1,4}$ Gogce Crynen,,3 Corbin Bachmeier, ${ }^{1-3}$ Janice Stewart, ${ }^{5}$ \\ Michael Mullan, ${ }^{1-3}$ William Stewart, ${ }^{5,6}$ and Fiona Crawford ${ }^{1-3}$
}

\begin{abstract}
Concussion or mild traumatic brain injury (mTBI) represents the most common type of brain injury. However, in contrast with moderate or severe injury, there are currently few non-invasive experimental studies that investigate the cumulative effects of repetitive mTBI using rodent models. Here we describe and compare the behavioral and pathological consequences in a mouse model of single (s-mTBI) or repetitive injury (r-mTBI, five injuries given at $48 \mathrm{~h}$ intervals) administered by an electromagnetic controlled impactor. Our results reveal that a single mTBI is associated with transient motor and cognitive deficits as demonstrated by rotarod and the Barnes Maze respectively, whereas r-mTBI results in more significant deficits in both paradigms. Histology revealed no overt cell loss in the hippocampus, although a reactive gliosis did emerge in hippocampal sector CA1 and in the deeper cortical layers beneath the injury site in repetitively injured animals, where evidence of focal injury also was observed in the brainstem and cerebellum. Axonal injury, manifest as amyloid precursor protein immunoreactive axonal profiles, was present in the corpus callosum of both injury groups, though more evident in the r-mTBI animals. Our data demonstrate that this mouse model of mTBI is reproducible, simple, and noninvasive, with behavioral impairment after a single injury and increasing deficits after multiple injuries accompanied by increased focal and diffuse pathology. As such, this model may serve as a suitable platform with which to explore repetitive mTBI relevant to human brain injury.
\end{abstract}

Key words: behavior; immunohistochemistry; in vivo studies; models of injury; TBI

\section{Introduction}

I T IS ESTIMATED THAT 1,700,000 traumatic brain injuries (TBI) occur annually in the United States with $\sim 75 \%$ of these categorized as mild TBI (mTBI) or concussion. ${ }^{1}$ Since the 1970s, several studies have reported a relationship between repetitive brain injury and progressive neurological deterioration. ${ }^{2-7}$ However, it is only recently that mTBI has become a major health issue, in part because of increased media attention surrounding the frequency and outcome of repetitive mTBI (r-mTBI) in military personnel and professional athletes. ${ }^{8-14}$ One of the earliest descriptions of the neurological consequences of brain injury characterized the neuropathological features associated with boxing in the condition originally termed "dementia pugilistica.", More recently, similar pathology, now referred to as chronic trau- matic encephalopathy (CTE), has been reported in athletes who sustain repeated mild concussions. ${ }^{15,16}$ There is growing recognition that for both repetitive and single moderate to severe injuries, the adverse effects of TBI may continue for many years after the original event, with trauma representing the strongest environmental risk factor for developing neurodegenerative disorders, including Alzheimer's disease. ${ }^{12,17-19}$

Studying mild brain injury in humans is challenging, as it is restricted to cognitive assessment and brain imaging to evaluate such injuries. Although the full complexity of human brain injury cannot be completely addressed in laboratory models, they offer the ability to investigate molecular and neurophysiological changes from minutes to days following nonfatal injury. Different animal models that have been studied to characterize the consequences of TBI include pigs, ${ }^{20-22}$ and primates, ${ }^{23}$ but primarily these studies

\footnotetext{
${ }^{1}$ Roskamp Institute, Sarasota, Florida.

${ }^{2}$ James A. Haley Veterans Administration Medical Center, Tampa, Florida.

${ }^{3}$ The Open University, Department of Life Sciences, Milton Keynes, United Kingdom.

${ }^{4}$ University of Cardiff, School of Biosciences, Cardiff, United Kingdom.

${ }^{5}$ Department of Neuropathology, Institute of Neurological Sciences, Southern General Hospital, Glasgow, United Kingdom.

${ }^{6}$ University of Glasgow, Department of Neuropathology, Glasgow, United Kingdom.
} 
have been performed using rodents. ${ }^{24-31}$ This report characterizes the neuropathological and neurobehavioral consequences of both s-mTBI and r-mTBI in a novel closed head injury (CHI) mouse model of concussion; our choice of a murine model driven by the future potential to study this mTBI model in mice genetically modified at loci of relevance to TBI such as APOE or Tau. The CHI model used in this study is more representative of mTBI/concussive conditions than invasive brain injury models such as fluid percussion injury (FPI) or controlled cortical impact $(\mathrm{CCI})$ that require a craniectomy, which itself can confer profound proinflammatory and behavioral damages. ${ }^{32}$ It is also amenable to the study of repeated concussions and, in terms of reproducibility, incorporates an electromagnetic (EM) coil-based delivery device, which delivers consistent strike velocities. ${ }^{33,34}$

To date, published mouse models of $\mathrm{CHI}$ have typically investigated the effect of a single mTBI or two mTBIs with an interinjury interval of $24 \mathrm{~h}^{24,26,31,35}$ A single mTBI was shown to cause subtle and transient behavioral and immunohistochemical abnormalities, whereas two such injuries $24 \mathrm{~h}$ apart worsened the outcome $\mathrm{e}^{24,26,27,31}$ Unfortunately, little is known about the cumulative consequence of more than two brain injuries at multiple time intervals, as investigations of this nature are scarce and have predominantly employed the weight drop model. ${ }^{25,28,36,37}$ In this study, we addressed this void by examining mice subjected to a s-mTBI or to a total of five r-mTBIs. Moreover, our interconcussive interval was $48 \mathrm{~h}$, a temporal window during which the mouse brain is known to be vulnerable to subsequent injuries ${ }^{27}$ in order to mimic human situations (combat or sports) in which additional injuries are sustained prior to full recovery from the previous injury. The purpose of these studies was to develop a reliable and robust mouse model of $\mathrm{mTBI} /$ concussion to investigate the neurobehavioral and neuropathological consequences of single and r-mTBI, and provide a platform for investigation of the long-term consequences of mTBI, particularly r-mTBI.

\section{Methods}

\section{Animals}

Male, C57BL/6J mice (10 weeks, 24-30g, Jackson Laboratories, Bar Harbor, ME) were singly housed under standard laboratory conditions $\left(23^{\circ} \mathrm{C} \pm 1{ }^{\circ} \mathrm{C}, 50 \% \pm 5 \%\right.$ humidity, and $12 \mathrm{~h}$ light/dark cycle) with free access to food and water throughout the study. Mice were allowed to adapt to the vivarium for 1 week prior to experimental procedures. All procedures involving mice were performed under Institutional Animal Care and Use Committee approval and in accordance with the National Institute of Health Guide for the Care and Use of Laboratory Animals.

\section{Injury groups and schedule}

For the behavioral analyses, a total of 48 mice were randomly assigned to one of four groups: single injury, single sham (anesthesia alone), repetitive injury (total of five hits with an interconcussion interval of $48 \mathrm{~h}$ ), and repetitive sham (five anesthesias, $48 \mathrm{~h}$ apart). The behavior analysis began $24 \mathrm{~h}$ after the sole/last mTBI/anesthesia for each group (Fig. 1). Behavior outcomes were assessed by an experimenter blinded to group assignment.

For histological examination, a total of 35 mice were randomly assigned to one of five treatment groups: single injury, single sham, repetitive injury, repetitive sham (all euthanized at $24 \mathrm{~h}$ post sole/ last $\mathrm{mTBI} /$ anesthesia), and single injury euthanized at 10 days post mTBI (s-mTBI-10D). The s-mTBI-10D treatment group was included to enable comparison of the consequences of one hit versus five hits at the same time point after the first injury.

\section{Injury protocol}

Mice were anesthetized with $1.5 \mathrm{~L} / \mathrm{min}$ of oxygen and $3 \%$ isoflurane and, after its head was shaved, each mouse was transferred into a stereotaxic frame (Just For Mice ${ }^{\mathrm{TM}}$ Stereotaxic, Stoelting, Wood Dale, IL) mounted with an EM controlled impact device (Impact One ${ }^{\mathrm{TM}}$ Stereotaxic Impactor, Richmond, IL). The animals were placed on a heating pad to maintain their body temperature at $37^{\circ} \mathrm{C}$ and noninvasive rubber pads were adjusted in height to level the skull. The head holders were positioned such that lateral movements would not occur when the head was impacted. A $5 \mathrm{~mm}$ blunt metal impactor tip was retracted and positioned above the sagittal suture midway before each impact (Fig. 2). The injury was triggered using the myNeuroLab controller at a strike velocity of $5 \mathrm{~m} / \mathrm{sec}$, strike depth of $1.0 \mathrm{~mm}$, and dwell time of $200 \mathrm{~ms}$; these parameters selected after pilot experiments revealed that a strike velocity $>5 \mathrm{~m} / \mathrm{sec}$ and/or strike depth $>1.0 \mathrm{~mm}$ resulted in skull fracturing. Based on the manufacturer's instructions, the force applied to the mouse head at the time of impact is $72 \mathrm{~N}$ under these conditions. At the end of the procedure, the animal was removed from the stereotaxic table and allowed to recover on a heating pad and, upon becoming ambulatory, was returned to its home cage. For the r-mTBI group, additional injuries were administered at days 3, 5,7 , and 9 after the original injury. Sham injured animals underwent the same procedures and anesthesia duration on each occasion, but did not receive a hit, in order to control for the effects of repeated anesthesia.

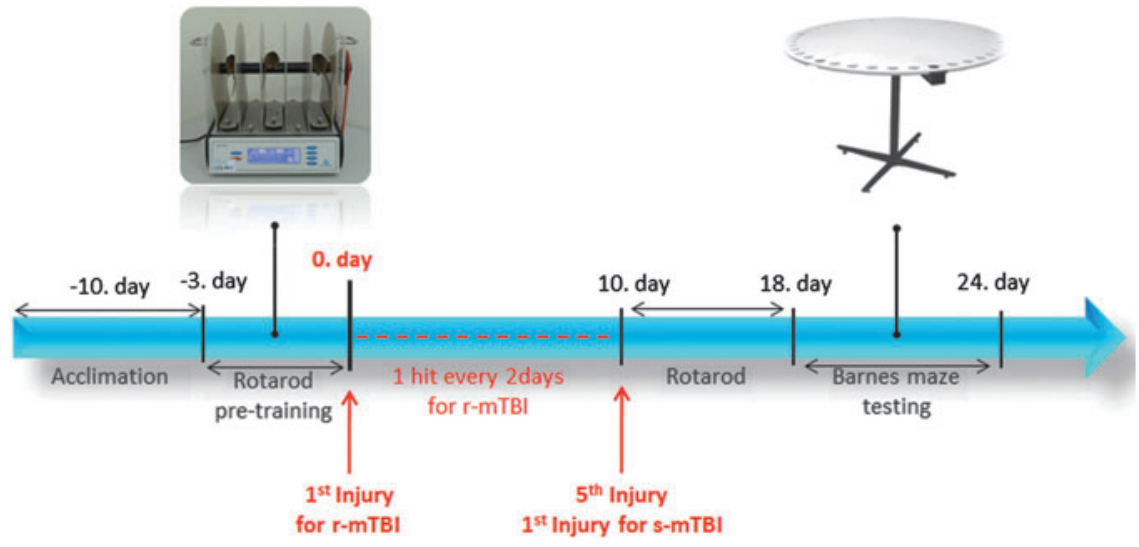

FIG. 1. Outline of experimental schedule. Color image is available online at www.liebertonline.com/neu 


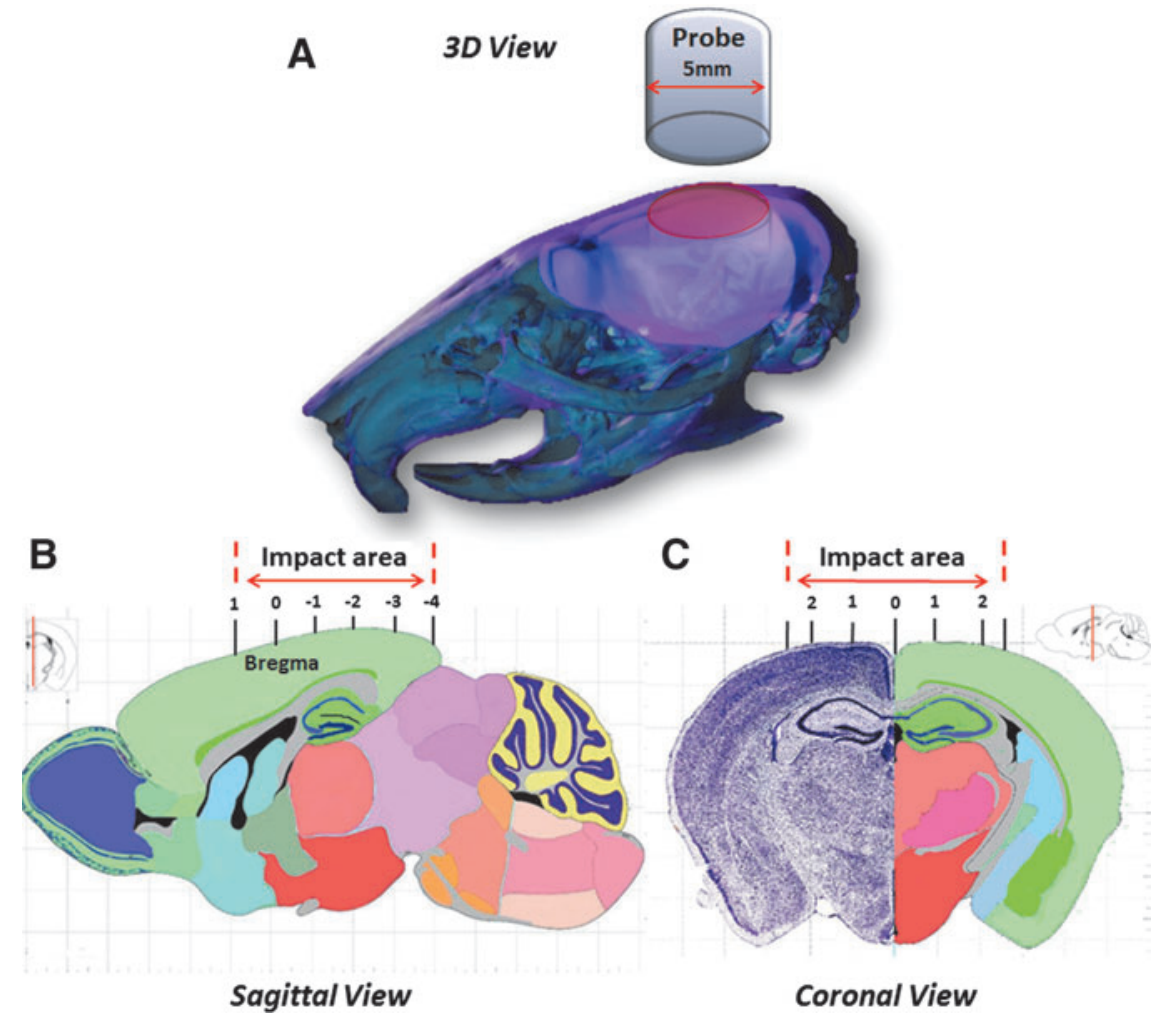

FIG. 2. Stereotaxic location of the $5 \mathrm{~mm}$ impactor tip on the head surface. Three-dimensional view (A) (image adapted with permission from Stephen Larson http://wholebraincatalog.org), sagittal view (B), and coronal view (C) (Images adapted from Franklin et al., 2001). ${ }^{38}$ Color image is available online at www.liebertonline.com/neu

\section{Assessment of righting reflex and traumatic apnea}

Immediately after termination of anesthesia of both impacted and sham groups, each animal was placed on its back and the latency period to return to an upright position was recorded. Apnea duration was visually measured after each injury as the time to return to spontaneous breathing. The time taken by injured and sham animals to return to an upright position and the traumatic respiration depression following each injury was recorded as an indicator of restoration of neurological function.

\section{Assessment of motor function}

The rotarod apparatus (Ugo Basile, Varese, Italy) was used to assess motor performance following the injury paradigm, with performance evaluated by measuring the latency to remain on an elevated rotating accelerating rod $(3 \mathrm{~cm}$ in diameter). Three acclimation trials were performed at a fixed speed of 5 revolutions per minute (RPM) with a 3 min duration. Rotarod pre-training (to establish a performance baseline) was conducted at linear accelerating speed of 5-50 RPM for three trials per day for 3 consecutive days. Each trial lasted a maximum of 5 min with a 3 min rest interval to avoid fatigue. Rotarod testing started $24 \mathrm{~h}$ following the sole/last mTBI or anesthesia. For each trial, latency to fall was recorded by an experimenter blinded to group assignment. The trial was also terminated if the animal spun around the rod through three complete revolutions. Testing occurred on days 1, 3, 5, and 7 after the sole/last mTBI/anesthesia.

\section{Assessment of cognitive function}

After the final day of rotarod testing, cognitive function was evaluated using the Barnes Maze (BM). Ethovision XT (Noldus, Wageningen, the Netherlands) was used to track and record the movement of each animal. Mice were given $90 \mathrm{sec}$ to locate and enter the target box, and they were required to remain in the target box for $30 \mathrm{sec}$ prior to retrieval, regardless of success. For a period of 6 days, four trials were given per day, with mice starting from one of four cardinal points on each trial. On the 7th day, a single probe trial lasting $60 \mathrm{sec}$ was performed with the mouse starting from the center of the maze and the target box removed. An Ethovision XT system was used to continually record the position of the mouse and measure the distance from the target box 30 times per second for the duration of each trial. The sum of that value was expressed as cumulative distance from target hole. Escape latency was measured as the time taken for the mouse to enter the box.

\section{Histology}

All mice were euthanized $24 \mathrm{~h}$ after their last injury/sham injury except for one group that was euthanized 10 days after a single mTBI. All mice were anesthetized with isoflurane perfused transcardially with heparinized PBS, pH-7.4 followed by PBS containing $4 \%$ paraformaldehyde. After perfusion, the brains were postfixed in a solution of $4 \%$ paraformaldehyde at $4^{\circ} \mathrm{C}$ for $48 \mathrm{~h}$. The intact brains were then blocked and processed in paraffin using Tissue-Tek VIP (Sakura, USA). Sagittal ( $n=4$ brains/group) and coronal ( $n=3$ brains/group) $6 \mu \mathrm{m}$ sections were cut with a microtome (2030 Biocut, Reichert/Leica, Germany) and mounted on positively charged glass slides (Fisher, Superfrost Plus). Prior to staining, sections were deparaffinized in xylene, and rehydrated in an ethanol to water gradient. For each group, sets of sagittal (lateral $0.2-0.4 \mathrm{~mm})$ and coronal $(-1.5$ and $-3.0 \mathrm{~mm}$ relative to bregma) sections were cut. Each slide was visualized with a bright field microscope (Leica, Germany) and digital images were scanned without zoom and with a resolution of $16896 \times 26624$ pixels for further analysis with a NanoZoomer (Hamamatsu, Japan). The slides were viewed using Slidepath Digital Image Hub software. 
Sections were stained with hematoxylin and eosin (H\&E) and combined Luxol fast blue and cresyl violet (LFB/CV) using standard histological protocols. Sets of adjacent sections were stained for glial fibrillary acid protein (GFAP, 1:20,000; Dako, Glostrup, Denmark, ZO334), ionized calcium binding adaptor molecule 1 (Anti-Iba1. 1:5000; Abcam, Cambridge, MA, ab5076), or amyloid precursor protein (APP, 1:40,000; Millipore, Billerica, MA, MAB348). As a negative control, one section was incubated with all reagents except the primary antibody. Tissue sections were subjected to antigen retrieval with either heated tris-ethylenediaminetetraacetic acid (EDTA) buffer ( $\mathrm{pH}-8.0)$ or modified citrate buffer (Dako, Glostrup, Denmark, S1699) under pressure. Endogenous peroxidase activity was quenched with a 15 min $\mathrm{H}_{2} \mathrm{O}_{2}$ treatment (3\% in water). Each section was rinsed and incubated with the appropriate blocking buffer (ABC Elite kit, MOM kit, Vector Laboratories, CA) for $20 \mathrm{~min}$, before applying the appropriate primary antibody overnight at $4{ }^{\circ} \mathrm{C}$. Then, the diluted biotinylated secondary antibody from the ABC Elite Kit was applied on each glass slide. Antibodies were detected using the avidin-peroxidase complex, and labeling was revealed after incubating the sections in 3,3'-diaminobenzidine (DAB) peroxidase solution (0.05\% DAB - $0.015 \% \mathrm{H}_{2} \mathrm{O}_{2}$ in $0.01 \mathrm{M}$ PBS, $\mathrm{pH}$ 7.2) for $6-7 \mathrm{~min}$ and counterstained with hematoxylin.

\section{Immunohistochemical quantification}

For each animal, sets of sagittal $(n=4)$ and coronal $(n=3)$ sections were stained and analyzed by an observer blinded to experimental conditions using ImageJ software (US National Institutes of Health, Bethesda, MD). Using this software, images were separated into individual color channels (hematoxylin counter stain and DAB chromagen) using the color deconvolution algorithm. ${ }^{39}$ Two non-overlapping areas of $200 \mu \mathrm{m}^{2}$ in the CA1 region and two non-overlapping areas of $150 \mu \mathrm{m}^{2}$ in the corpus callosum (CC) were randomly selected within which the area of GFAP immunoreactivity was calculated and expressed as a percentage of the field of view. Two non-overlapping areas of $200 \mu \mathrm{m}^{2}$ in the cortex underlying the impact site and three non-overlapping areas of $100 \mu \mathrm{m}^{2}$ in the CC were randomly selected within which the area of anti-Iba1 immunoreactivity was calculated and expressed as a percentage of the field of view. APP-immunohistochemistry was quantified in discrete areas, namely the CC and the brainstem (BS) (lateral $0.2-0.4 \mathrm{~mm}$ ). The numbers of APP-positive profiles were counted in three non-overlapping areas of $100 \mu \mathrm{m}^{2}$ within the CC, and two non-overlapping areas of $200 \mu \mathrm{m}^{2}$ within the BS. Immunoreactive axonal profile counts from the four sections from each animal $(n=4)$ were then averaged, and all were combined for each group to determine a mean value. Overt cell death was determined by assessing the density of degenerating neurons (acidophilic with shrunken perikarya and pyknotic nuclei) per $\mu \mathrm{m}^{2}$ in H\&E stained sections (lateral $0.2-0.4 \mathrm{~mm}$ ). The percentage of degenerating neurons in CA1 regions in the ipsilateral and contralateral hippocampus was then averaged across four sections to evaluate histological changes in each group.

\section{Statistical analysis}

All data were analyzed using JMP 8.0 (SAS, Cary, NC). Data were tested for normality using the Shapiro-Wilk W test; when not normally distributed, the data were transformed using square root or natural $\log$ transformation. If the data were still not normal after transformation, they were analyzed using nonparametric methods. Normally distributed data were analyzed using parametric methods such as analysis of variance (ANOVA) and $t$-test. Repeated measures ANOVA was used to compare performance between cohorts for both rotarod and BM experiments. Only $p$ values $<0.05$ were considered to be statistically significant (indicated by an asterisk in the figures).

\section{Results \\ Acute neurological responses}

Following CHI, all injured animals demonstrated a period of apnea ranging from 3 to $30 \mathrm{sec}$ followed by a prolonged period of unresponsiveness (3-6 min). There were no significant differences among impact apneas across injury groups, although the time of apnea did decrease with each mTBI in the r-mTBI group (repeated measures ANOVA). In addition, there were no significant differences in righting between sham and injured groups. Similar to apnea, the righting reflex recovery duration slightly decreased with each mTBI, although this was not significant (repeated measures ANOVA). Therefore, regardless of the number of injuries, the traumatic apnea and the time required for recovery of the righting reflex was similar, and did not increase with increasing number of injuries.

\section{Sensorimotor function}

Motor function was evaluated $24 \mathrm{~h}$ following the sole/last mTBI/ anesthesia. Overall, all animals exhibited a decreased rotarod performance when compared to their last day of pre-training (Fig. 3). By the last day of rotarod, the s-mTBI and r-mTBI fall latency was

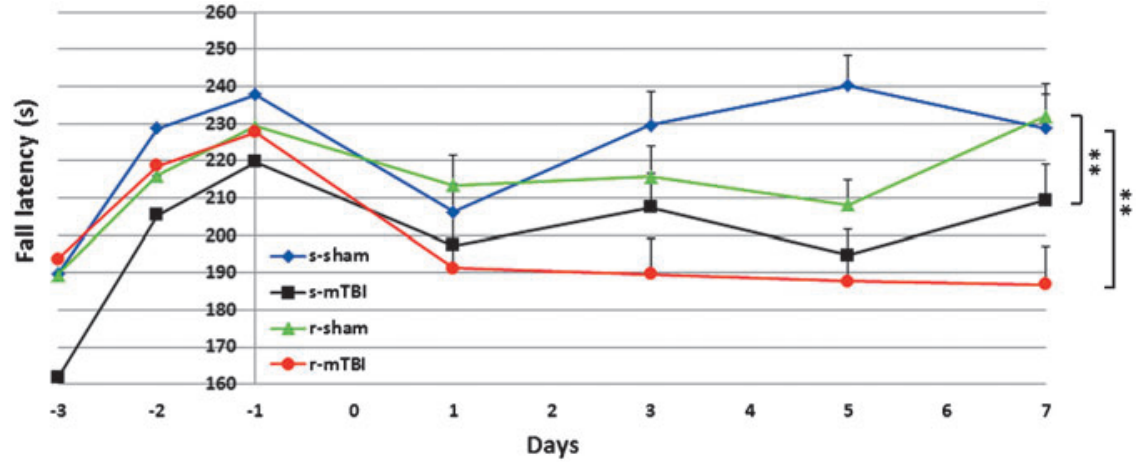

FIG. 3. Effect of mild traumatic brain injury (mTBI) on rotarod performance. Values were recorded on 7 separate days with three 5 min accelerating trials. Results are the mean \pm SEM of the time animals remained on the rotarod before falling. There was a gradation of impairment with multiply injured animals remaining less time on the rod between days 1 and 7 after sole/last mTBI or anesthesia (s-mTBI vs. s-sham [** $p=0.005$ ]; r-mTBI vs. r-sham [** $p<0.001$ ]; $n=12$ per group]). Color image is available online at www.liebertonline.com/neu 
shorter than that of their sham controls, $9 \%$ and $19 \%$ less, respectively (s-mTBI, $p=0.005$; r-mTBI, $p<0.001$; repeated measures ANOVA). Mice with a single mTBI improved over the testing period from $90 \pm 13 \%$ of pre-injury level on day 1 to $95 \pm 12 \%$ on day 7 (interaction "Injury and test day"; $p<0.05$; repeated measures ANOVA). In contrast, the r-mTBI mice did not return to an uninjured baseline level; $84 \pm 9 \%$ of pre-injury level on day 1 to $82 \pm 15 \%$ on day 7 (interaction "Injury and test day"; $p>0.05$; repeated measures ANOVA). Rotarod testing was also conducted at 24 and 30 days after the final injury, but by this extended time point the performance of singly or repetitively injured animals was not different from that of controls, demonstrating the transient nature of the motor deficits.

\section{Cognitive deficit after single and repetitive injury}

Acquisition deficits were observed in brain-injured mice relative to their sham controls (Fig. 4A and B). Again, we found that the repetitively injured animals were performing worse than the singly injured mice. Although both sham and injured mice were able to find the correct hole by the last day of the acquisition period, both singly and repetitively injured mice had a greater cumulative distance from the hole than their sham controls (52\% and $73 \%$ further, respectively) (s-mTBI, $p<0.005$; r-mTBI, $p<0.0001$; repeated measures ANOVA). Their ability to find a previously learned location of the escape hole was also assessed and all groups showed improvement in escape latency, but with the r-mTBI group performing worse than controls and the s-mTBI group. Only day 6 from this dataset passed normality testing, and analyzing that day alone, the r-mTBI group was found to be significantly different from the other three groups $(p<0.0001)$. The velocity across each group was similar, (s-sham, $6.3 \pm 0.34 \mathrm{~cm} / \mathrm{s}$; $\mathrm{r}$-sham, $6.6 \pm 0.60 \mathrm{~cm} / \mathrm{s} ; \mathrm{s}-\mathrm{mTBI}, 5.9 \pm 0.34 \mathrm{~cm} / \mathrm{s} ; \mathrm{r}-\mathrm{mTBI}, 6.4 \pm$ $0.31 \mathrm{~cm} / \mathrm{s}$ ) indicating that differences in $\mathrm{BM}$ performance were not a function of motor deficits.

The probe trial analysis of the average time to reach the target zone, (defined by the target escape hole and its adjacent north and south holes) revealed that the r-mTBI mice performed the worst, requiring on average $15 \mathrm{sec}$ to reach the target zone, followed by the s-mTBI $(8.3 \mathrm{sec})$, the r-sham $(4.8 \mathrm{sec})$, and the s-sham $(2.9 \mathrm{sec})$. For both injury groups, the time to reach the target or adjacent holes was significantly longer than for their sham controls $(p<0.02$ for both injury groups; ANOVA) (Fig. 4C). As with the acquisition trials, the average velocity was not significantly different across all four groups $(p>0.05)$.

\section{Pathology of single and repetitive injury}

Macroscopic examination of fixed brains revealed focal architectural disturbance with evidence of hemorrhage $\left(<1 \mathrm{~mm}^{2}\right)$ in the inferior surface of the cerebellum in all animals subjected to r-mTBI. These were also evident, but to a lesser extent, in every singly injured mouse at 10 days after injury. Otherwise, there were no focal macroscopic lesions in any of the animals subjected to injury. Specifically, there were no skull fractures, cerebral hemorrhages, or contusions identified using this injury model. Further, examining sections stained for $\mathrm{H} \& \mathrm{E}$ or $\mathrm{LFB} / \mathrm{CV}$ revealed no evidence of focal structural pathology in the cortex, hippocampus, or hilus of the dentate gyrus (Fig. 5), with quantification of hippocampal neurons demonstrating no overt cell loss in either the ipsi- or contralateral hippocampi of the injured groups. Animals subjected to s-mTBI displayed no evidence of cerebellar injury at $24 \mathrm{~h}$ post- injury (Fig. 5F). However, at 10 days after single injury, a
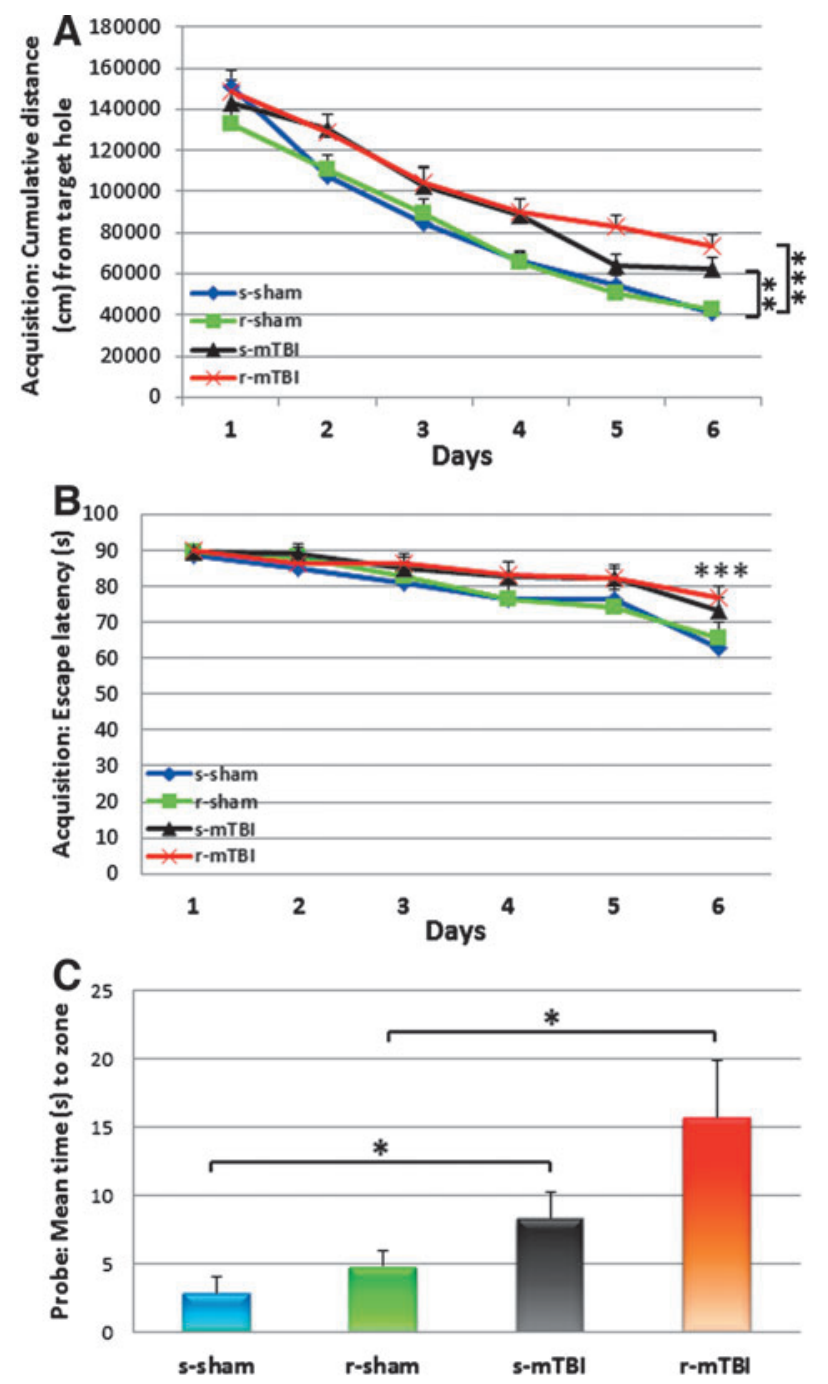

FIG. 4. Evaluation of learning (acquisition) and spatial memory retention (probe) using the Barnes maze on days 8-14 after sole/ last mild traumatic brain injury (mTBI). Mice were tested in the Barnes maze for their ability to locate a black box at the target hole. During the acquisition testing, both injured groups traveled a greater cumulative distance before escaping to the target hole compared with their respective anesthesia controls, with the $r$-mTBI groups versus the $r$-sham group showing a greater significant difference than the s-mTBI groups versus the s-sham group (**s-mTBI, $p<0.005$; r-mTBI, $* * * p<0.0001)(\mathbf{A})$. The mean time to escape to the target hole was longer for the r-mTBI group. On day 6 of the acquisition trial, the r-mTBI group spent significantly more time escaping to the target hole than did their sham control, $\mathrm{r}$-sham $(* * * p<0.0001)(\mathbf{B})$. For the probe trial (1 day following the 6 days of acquisition testing), the target box was removed and mice were placed in the middle of the table for a single, $60 \mathrm{sec}$ trial. Although both mTBI groups had a greater latency to reach the target zone than their respective shams $\left({ }^{*} p<0.02\right)$, there was a clear trend for the r-mTBI mice to take longer to reach the target zone $(\mathbf{C})$. The mean velocity for acquisition phase and probe test was similar across all groups (data not shown). Data are presented as mean \pm SEM. Color image is available online at www.liebertonline.com/neu

focal, superficial region of architectural disruption associated with a glial response was observed in the inferior surface of the cerebellum (Fig. 5I). In the r-mTBI group, this lesion was extensive and extended into underlying white matter (Fig. 5O). 

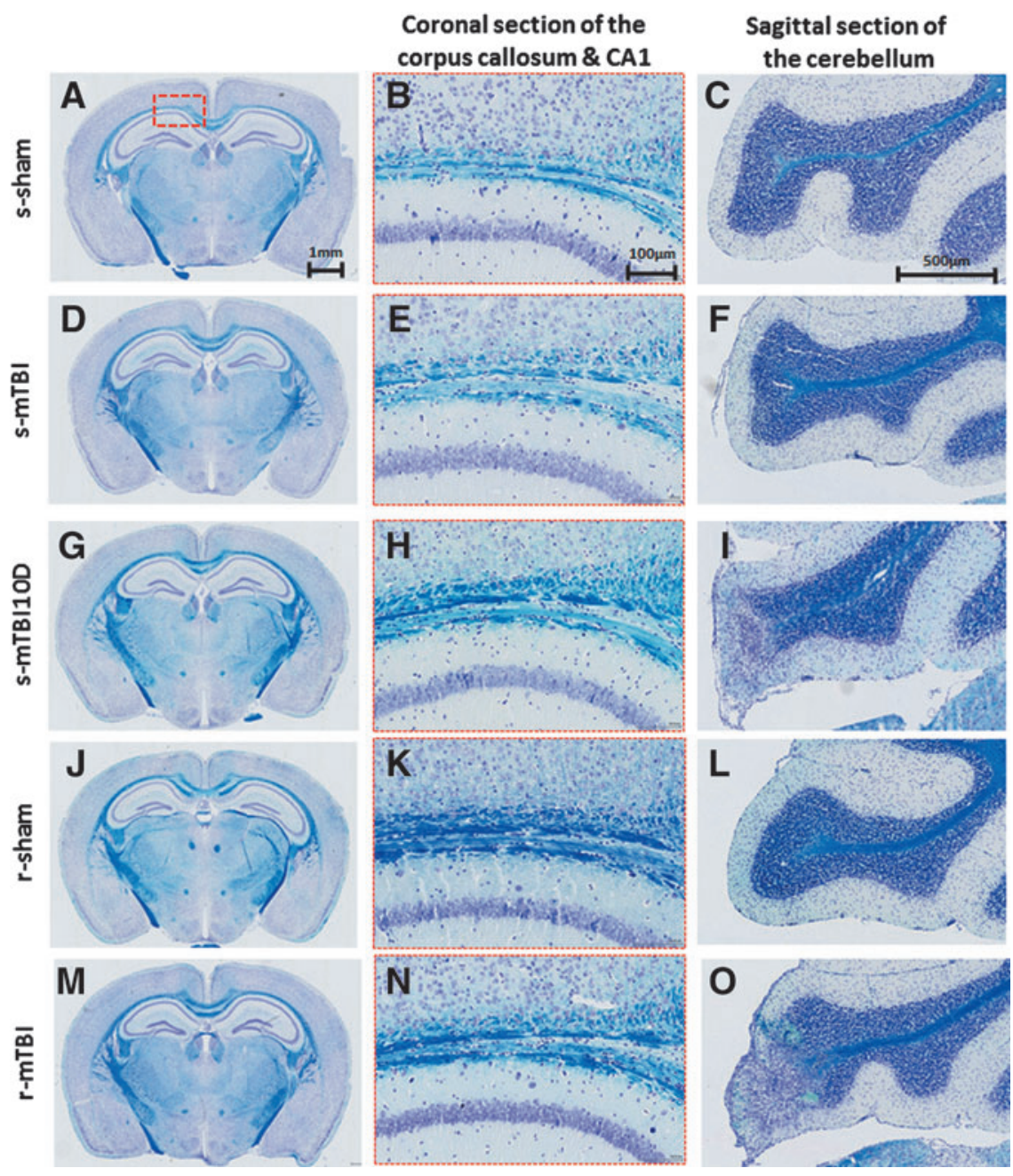

FIG. 5. Luxol fast blue/cresyl violet staining revealed no overt abnormalities in the cerebral cortex of the injured or non-injured mice. Coronal sections of mouse brain at $\sim 1.9 \mathrm{~mm}$ posterior to bregma $(\mathbf{A}, \mathbf{D}, \mathbf{G}, \mathbf{J}, \mathbf{M})$. The red box indicates the region of interest, which is shown at a higher magnification $(\mathbf{B}, \mathbf{E}, \mathbf{H}, \mathbf{K}, \mathbf{N})$. Sagittal sections of the cerebellum $(0.26 \mathrm{~mm}$ lateral to midline) $(\mathbf{C}, \mathbf{F}, \mathbf{I}, \mathbf{L}, \mathbf{O})$. A focal region of architectural disturbance in the inferior surface of the cerebellum was observed in the r-mTBI group (O), which was less extensive in the s-mTBI-10D group (I). Color image is available online at www.liebertonline.com/neu

\section{APP immunostaining}

Numerous APP-immunoreactive axonal profiles were identified in sections from injured animals (Fig. 6A and B). These APPimmunoreactive axonal profiles were observed as either granular or more elongated, fusiform swellings in the white matter of the parasagittal cortex, the $\mathrm{CC}$, and the spinal trigeminal tracts of the BS. Unique to the r-mTBI group, there was also evidence of cytoplasmic staining in neurons of the primary and secondary motor cortex (Fig. 6C). APP-immunoreactive axonal profiles were observed $24 \mathrm{~h}$ post-injury in the CC of the s-mTBI (Fig. 7G) and r-mTBI groups (Fig. 7S) but not in their controls (Fig. 7C-O). The numbers of APP-immunoreactive profiles in the $\mathrm{CC}$ of the $\mathrm{s}-\mathrm{mTBI}$ was greater than in the r-mTBI group (s-mTBI group $14.4 \pm 2.26$ vs. r-mTBI $6.0 \pm 0.8$ axonal profiles $/ 100 \mu \mathrm{m}^{2}$; $p<0.001$; Fig. 8A). Axonal damage in the BS was minimal in the $\mathrm{s}-\mathrm{mTBI}$, whereas greater numbers of punctate immunoreactive swellings were present in the r-mTBI group (s-mTBI $0.4 \pm 0.2$ vs. r-mTBI $5.2 \pm 1$ axonal profiles $/ 200 \mu \mathrm{m}^{2} ; p<0.001$; Fig. 8B). By 10 days post-mTBI, no immunoreactive profiles were observed after s-mTBI (Fig. 7J and K).

\section{Glial fibrillary acidic protein immunostaining}

For mice subjected to r-mTBI, immunostaining for GFAP revealed evidence of a mild reactive astrogliosis in regions of the cortex underlying the impact site (Fig. 9R), the CC (Fig. 9S), and the hippocampus (Fig. 9T). In contrast, no gliosis was observed in the cortex at the impact site in shams (Fig. 9B) or $24 \mathrm{~h}$ after a single injury (Fig. 9F). In the CC, the r-mTBI (Fig. 9S) and s-mTBI-10D (Fig. 9K) groups showed a notable increase in the area of GFAP immunoreactivity compared with their respective sham controls, with the magnitude of this increase greater in the r-mTBI than in the s-mTBI-10D group (s-sham $0 \%$ vs. s-mTBI-10D $1.8 \pm 0.7 \%$; $p<0.0001 ; \mathrm{r}$-sham $0.6 .1 \pm 0.2 \%$ vs. $\mathrm{r}-\mathrm{mTBI} 5.0 \pm 0.7 \% ; p<0.0001$; Fig. 10A). In the CA1 region, there was no evidence of increased immunoreactivity in mice subjected to s-sham (Fig. 9D), or in the singly injured animals at either time point (Fig. 9H-L), (s-sham $1.5 \pm 0.2 \%$ vs. s-mTBI $1.2 \pm 0.2 \%$, vs. s-mTBI-10D $1.9 \pm 0.3 \%$; $p>0.05$ ). By contrast, increased GFAP immunoreactivity was observed in the CA1 region of the r-mTBI group (Fig. 9T) compared with its sham (Fig. 9P), (r-mTBI $5.7 \pm 0.4 \%$ vs. r-sham $3.8 \pm 0.5 \%$; Fig.10B; $p<0.005)$. Interestingly, we also observed an increase in 

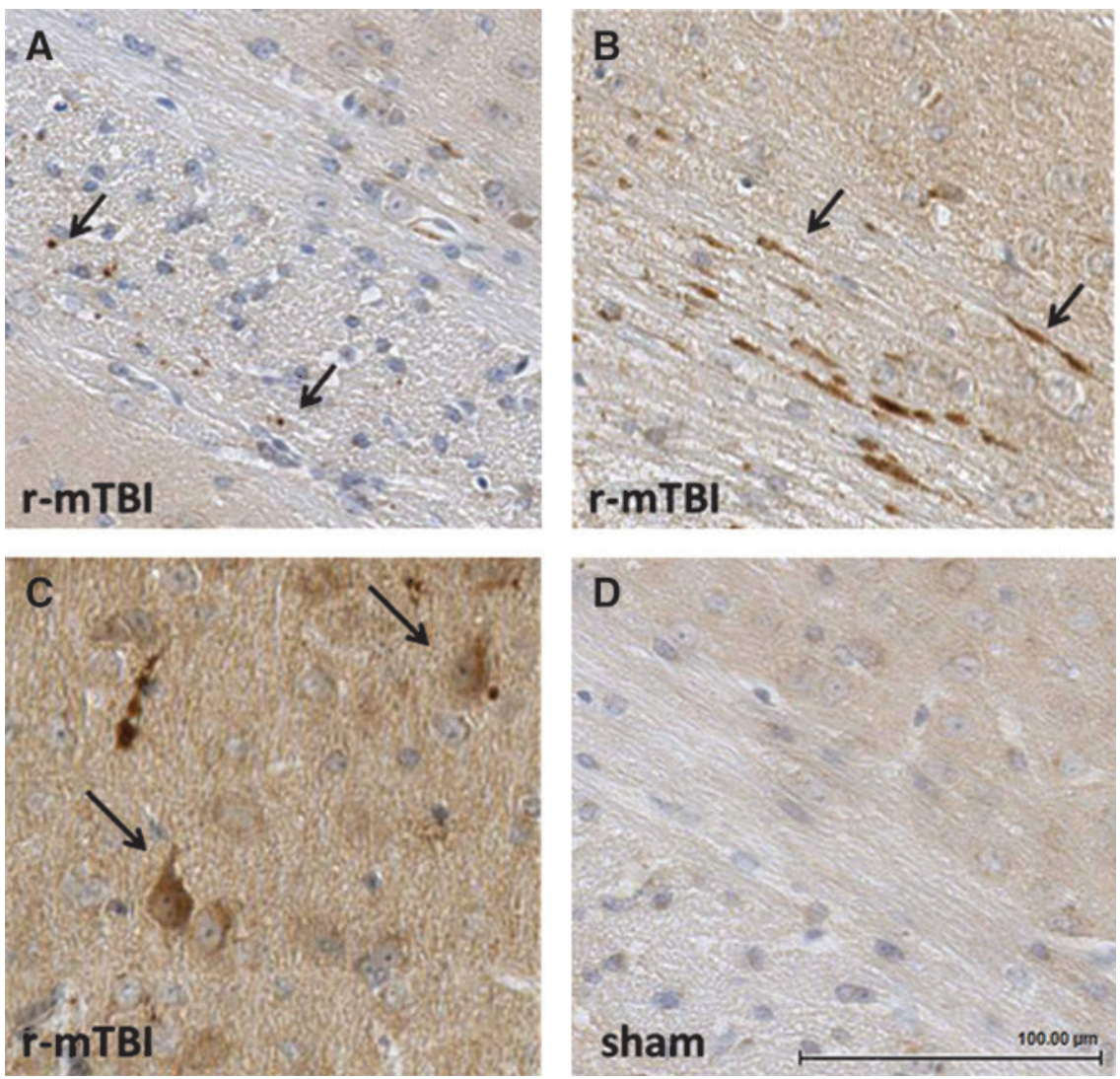

FIG. 6. Photomicrographs of sagittal sections stained with APP. Immunoreactive axonal profiles were observed as either granular (A) or more elongated, fusiform (B) swellings in both s-mTBI and r-mTBI groups (only the r-mTBI group is displayed). APP immunoreactive neurons were only present in the r-mTBI group, and occasionally observed in the cortex underneath the impact site (C). No APP staining was observed in the sham animals (D). Tissue sections were counterstained with hematoxylin. Scale bar, $100 \mu \mathrm{m}$. Color image is available online at www.liebertonline.com/neu

GFAP-immunoreactivity in the CA1 region of the r-sham animals when compared with the s-mTBI and the s-sham animals (Fig. 10B, $p<0.01)$.

\section{Iba1 immunostaining}

In the sham animals, none of the regions of interest showed cells with structural characteristics of activated microglia (hypertrophic and bushy morphology); (Fig. 11A-C; J-L). In the singly injury animals, microglial activation in the BS and in the cortex was not noticeable at either $24 \mathrm{~h}$ or 10 days post injury (Fig. 11D-I). However, in the CC, the s-mTBI and the s-mTBI-10D showed a notable increase in Iba1-immunoreactivity (s-sham $1.34 \pm 0.2 \%$ vs. s-mTBI $2.5 \pm 0.3 \%$, vs. s-mTBI-10D 2.9 $\pm 0.9 \% ; \quad p<0.005$; Fig.11E-H and Fig. 12A). For mice subjected to r-mTBI, immunostaining for anti-Iba-1 revealed clusters of activated microglias in the BS (Fig. $11 \mathrm{M}$ ), the CC (r-sham $1.5 \pm 0.3 \%$ vs. s-mTBI $8.5 \pm 0.8 \% ; p<0.0001$; Fig. $11 \mathrm{~N}$ ), and microglia, with a bushy morphology in the region of the cortex underlying the impact site (r-sham $0.3 \pm 0.09 \%$ vs. s-mTBI $4.0 \pm 1.1 \%$; $p<0.0001$; Fig. 110 ).

\section{Discussion}

We have developed and characterized a new mouse model of $\mathrm{CHI}$ produced by an EM controlled impact device. The purpose in developing this model is to establish an easily implemented, robust, and highly replicable animal model to address the consequences of mTBI and understand the cumulative and chronic effects of repetitive mild injury. Moreover, to our knowledge, this is the first study of its kind to examine the consequences of more than two repetitive hits at an interconcussive interval of $48 \mathrm{~h}$. Our results demonstrate that animals exposed to s-mTBI have short-term behavioral abnormalities that manifest as transient deficits in motor function and spatial memory, which are accompanied by reactive astrocytosis and sparse APP-immunoreactive axonal pathology in the CC. By contrast, animals subjected to r-mTBI with an interinjury interval of $48 \mathrm{~h}$ demonstrated greater cognitive impairment, microglial activation, more widespread and marked reactive astrocytosis, and multifocal axonal pathology, as well as focal injury in the cerebellum (see summary in Table 1).

The main limitation of this model is a function of interspecies physiological differences. The murine skull has a greater deformability than that of the rat, and its relatively small mass compared to that of humans complicates biomechanical studies, as it cannot simulate a true human rotational/angular injury induced by the brain inertia. ${ }^{40,41}$ Consequently, the lack of head movement is a common issue of brain concussion in subprimate animals, as this is inconsistent with human brain concussion. Nonetheless, the key elements of brain trauma neuropathology remain similar across species. For example, the pathological consequences of axonal injury (axonal varicosities, axonal bulbs) have been observed in humans, pigs, rats, and mice. ${ }^{17,22,31,42}$ The other advantages of mouse models (genetic manipulability, ease of protocol implementation, and cost) make this an appropriate platform in which to identify molecular consequences of repetitive versus single injury for exploration in higher mammals. 


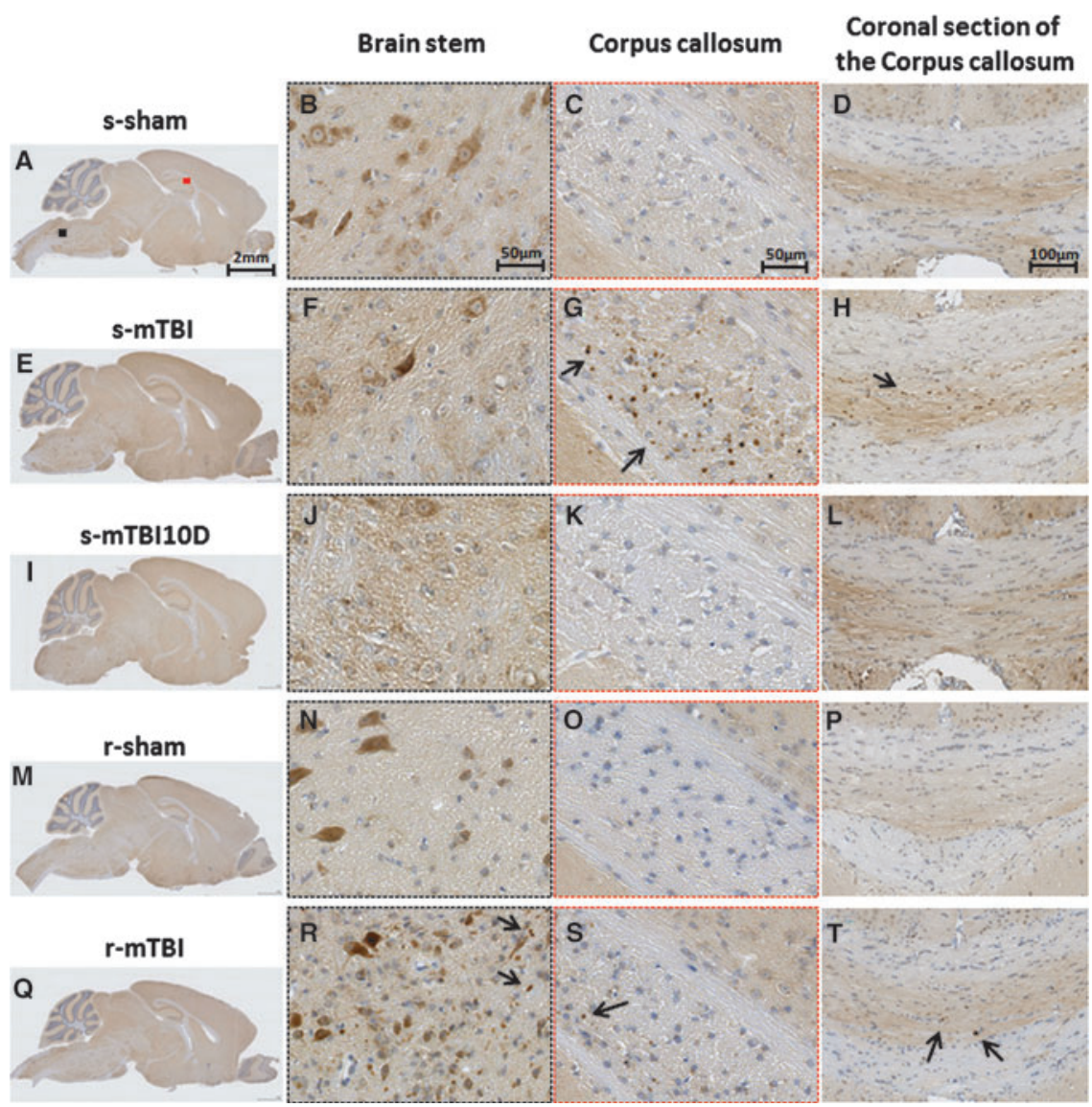

FIG. 7. Amyloid precursor protein immunohistochemistry of sagittal sections of mouse brain at $\sim 0.4 \mathrm{~mm}$ lateral to the midline in the brainstem $(\mathbf{B}, \mathbf{F}, \mathbf{J}, \mathbf{N}, \mathbf{R})$, in the corpus callosum $(\mathbf{C}, \mathbf{G}, \mathbf{K}, \mathbf{O}, \mathbf{S})$, and coronal sections $\sim 2.1 \mathrm{~mm}$ posterior to bregma $(\mathbf{D}, \mathbf{H}, \mathbf{L}, \mathbf{P}, \mathbf{T})$. Colored boxes indicate areas of interest shown at higher magnification in the right hand panels. Tissue staining from s-sham (A-D), s-mTBI10D (I-L), and r-sham animals (M-P) was negative for APP immunostaining. Immunoreactive axonal profiles were observed the brainstem in an area underlying the cerebellum in the r-mTBI $(\mathbf{R})$, whereas few such profiles were detected in the s-mTBI group $(\mathbf{F})$. Extensive accumulation of APP was observed as discrete axonal profiles in the corpus callosum of the s-mTBI $(\mathbf{G}, \mathbf{H})$, and less in the r-mTBI animals $(p<0.001)(\mathbf{S}, \mathbf{T})$. Tissue sections were counterstained with hematoxylin. Color image is available online at www.liebertonline.com/neu

A

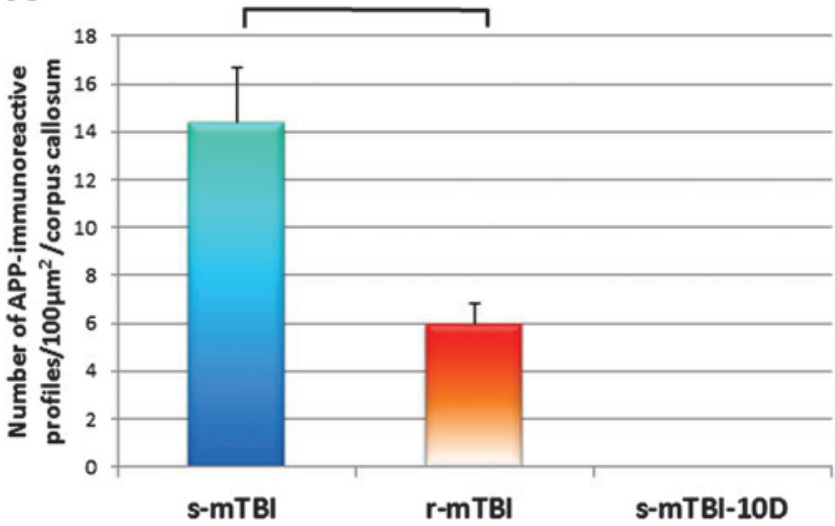

B

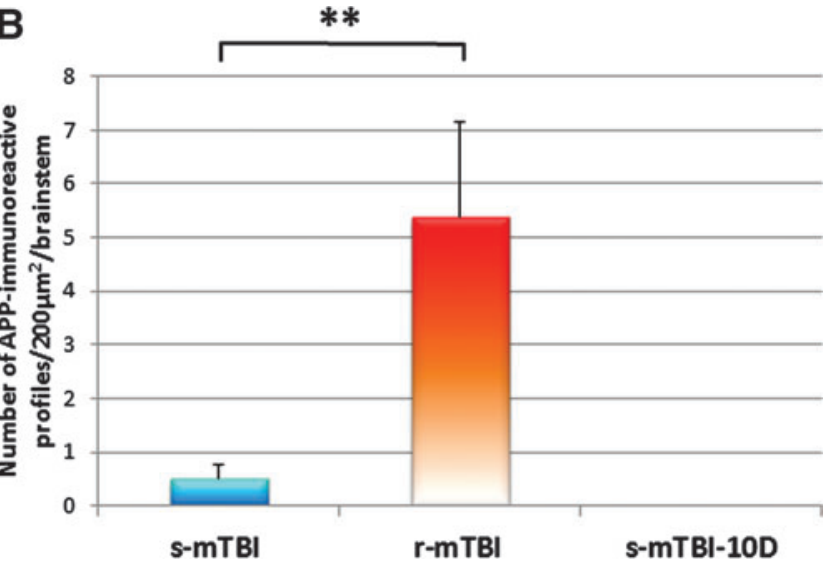

FIG. 8. Average counts of APP-immunoreactive axonal profiles per $\mu \mathrm{m}^{2}$ in the corpus callosum (A) and in the brainstem (B) $(n=4)$. Fewer axonal profiles were observed in the corpus callosum of the r-mTBI group than in the s-mTBI group ( $* * p<0.001)$. APPimmunoreactive axonal profiles the brainstem were observed in the r-mTBI group, whereas only a few were detected in the s-mTBI group $(* * p<0.001)$. Tissue staining from s-sham and s-mTBI10D animals was negative for APP immunostaining. Data are presented as mean \pm SEM. Color image is available online at www.liebertonline.com/neu 


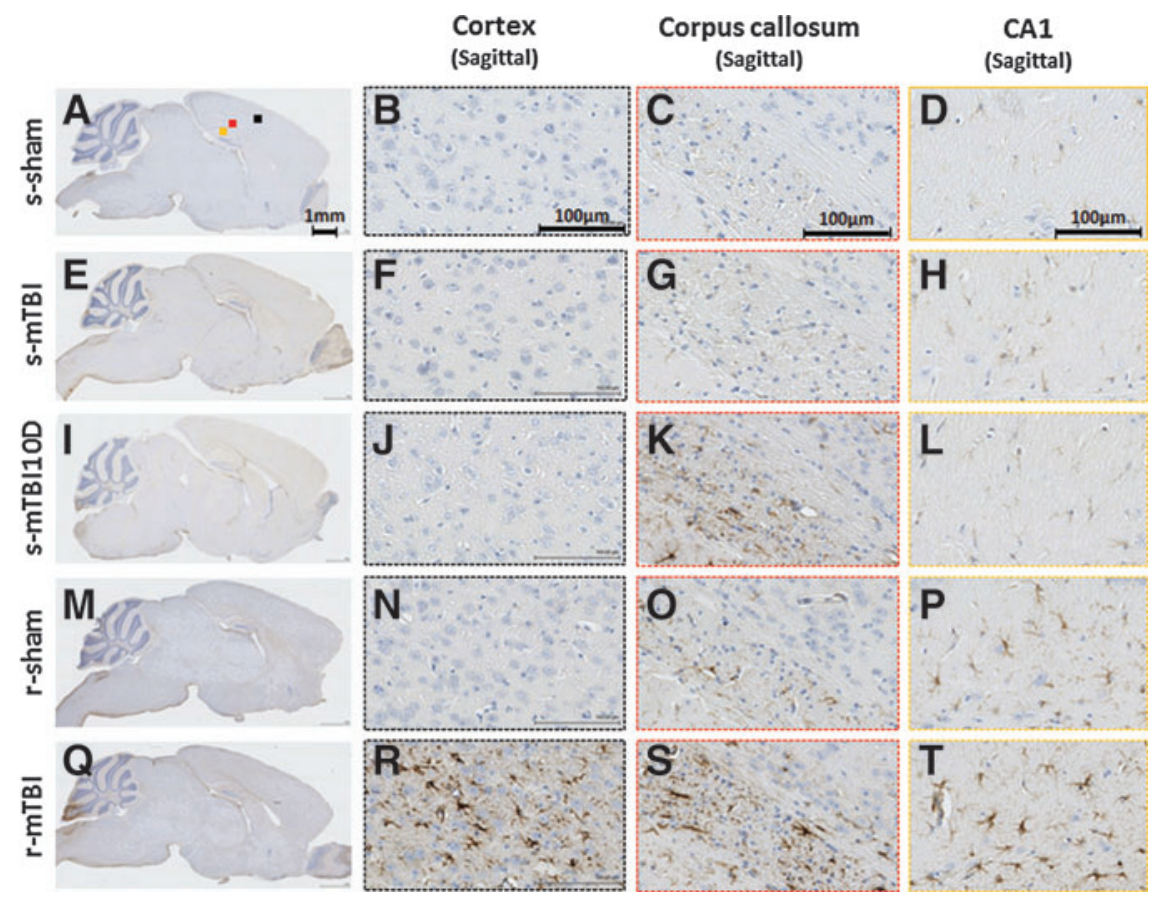

FIG. 9. Glial fibrillary acid protein (GFAP) immunohistochemistry of sagittal sections of the mouse brain at $\sim 0.4 \mathrm{~mm}$ lateral to midline in the cortex $(\mathbf{B}, \mathbf{F}, \mathbf{J}, \mathbf{N}, \mathbf{R})$, corpus callosum $(\mathbf{C}, \mathbf{G}, \mathbf{K}, \mathbf{O}, \mathbf{S})$, and the CA1 subregion of the hippocampus $(\mathbf{D}, \mathbf{H}, \mathbf{L}, \mathbf{P}, \mathbf{T})$. Colored boxes indicate areas of interest shown at higher magnification in the right panels. There were no changes in the s-mTBI group (E-H) compared with their respective sham group (A-D) at $24 \mathrm{~h}$ post-injury. An increase in the area of GFAP staining was observed in the corpus callosum at 10 days post s-mTBI $(\mathbf{K})$. An increased area of GFAP immunoreactivity was observed in the CA1 region of the $\mathrm{r}$-sham group (P). GFAP immunohistochemistry shows extensive staining in the r-mTBI at $24 \mathrm{~h}$ after final injury in the cortex, corpus callosum, and hippocampus (R-T). Tissue sections were counterstained with hematoxylin. Color image is available online at www.liebertonline.com/neu

Consistent with other mouse models of r-mTBI and evidence from athletes who have experienced repeated mild concussive injuries, our model shows acute postural equilibrium impairment post-injury as assessed by the rotarod motor task. ${ }^{37,43}$ Whereas the performance of the s-mTBI group improved over time, the r-mTBI group was unable to recover their sensorimotor function up to 7 days post-injury, which was the latest day tested in our studies. Over this time period, each injury group performed worse than their respective shams with a rank order of performance of $\mathrm{r}-\mathrm{mTBI}<$ $\mathrm{s}$-mTBI $<\mathrm{r}$-sham $=\mathrm{s}$-sham. When tested for cognitive performance in the BM, both injury groups showed cognitive impairment consistent with findings in previous brain injury studies. $25,30,31,42,44,45$

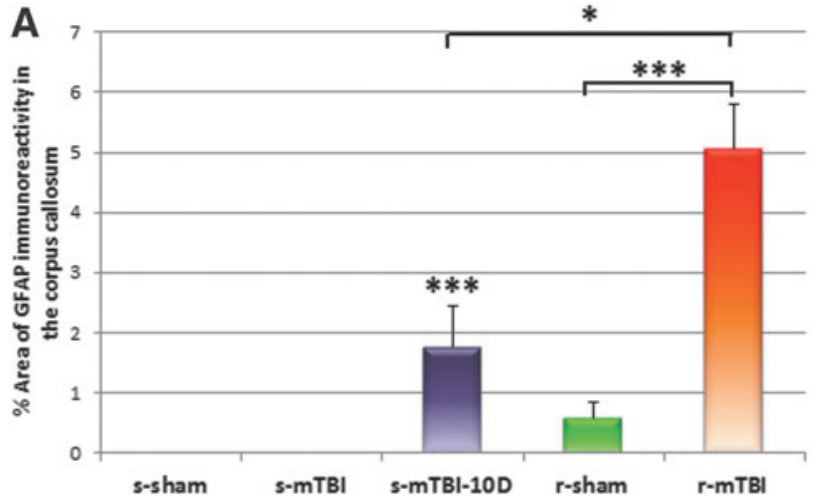

There was a worsening trend for r-mTBI compared with s-mTBI during the acquisition trials as well as on time to zone in the probe trial, and on the final day of acquisition training the r-mTBI group performed significantly worse than the other three groups.

From a neuropathological perspective, the only macroscopic abnormality observed was a focal contusion injury on the inferior surface of the cerebellum away from the site of injury in the r-mTBI and s-mTBI-10D groups. In addition, we observed an evolving cerebellar injury in the s-mTBI-10D group that is likely the result of secondary injury processes that develop over a period of days after the initial trauma. Aside from this observation, there was no evidence of structural damage to the brain based on routine

FIG. 10. Quantitative analysis of GFAP staining intensity in two $150 \mu \mathrm{m}^{2}$ areas of the corpus callosum (A) and two $200 \mu \mathrm{m}^{2}$ areas of the CA1 region (B) $(n=4)$. Data are presented as mean \pm SEM, $\left({ }^{*} p<0.05 ; * * p<0.001 ; * * * p<0.0001\right)$. Color image is available online at www.liebertonline.com/neu

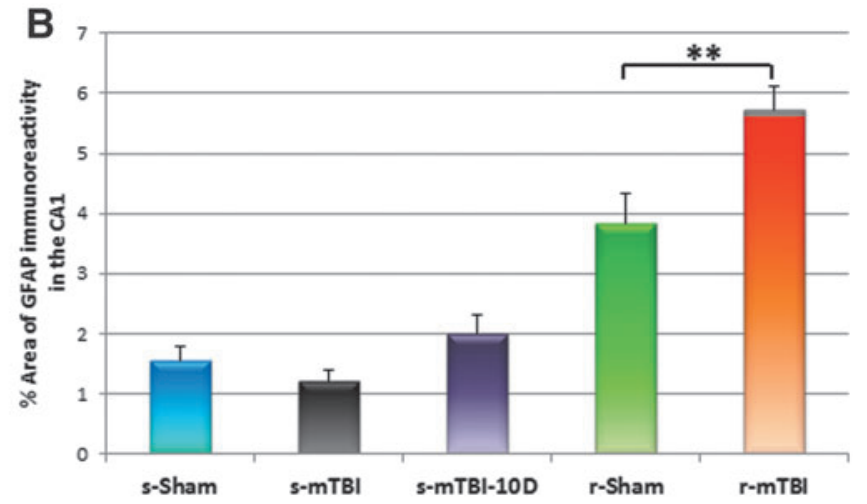



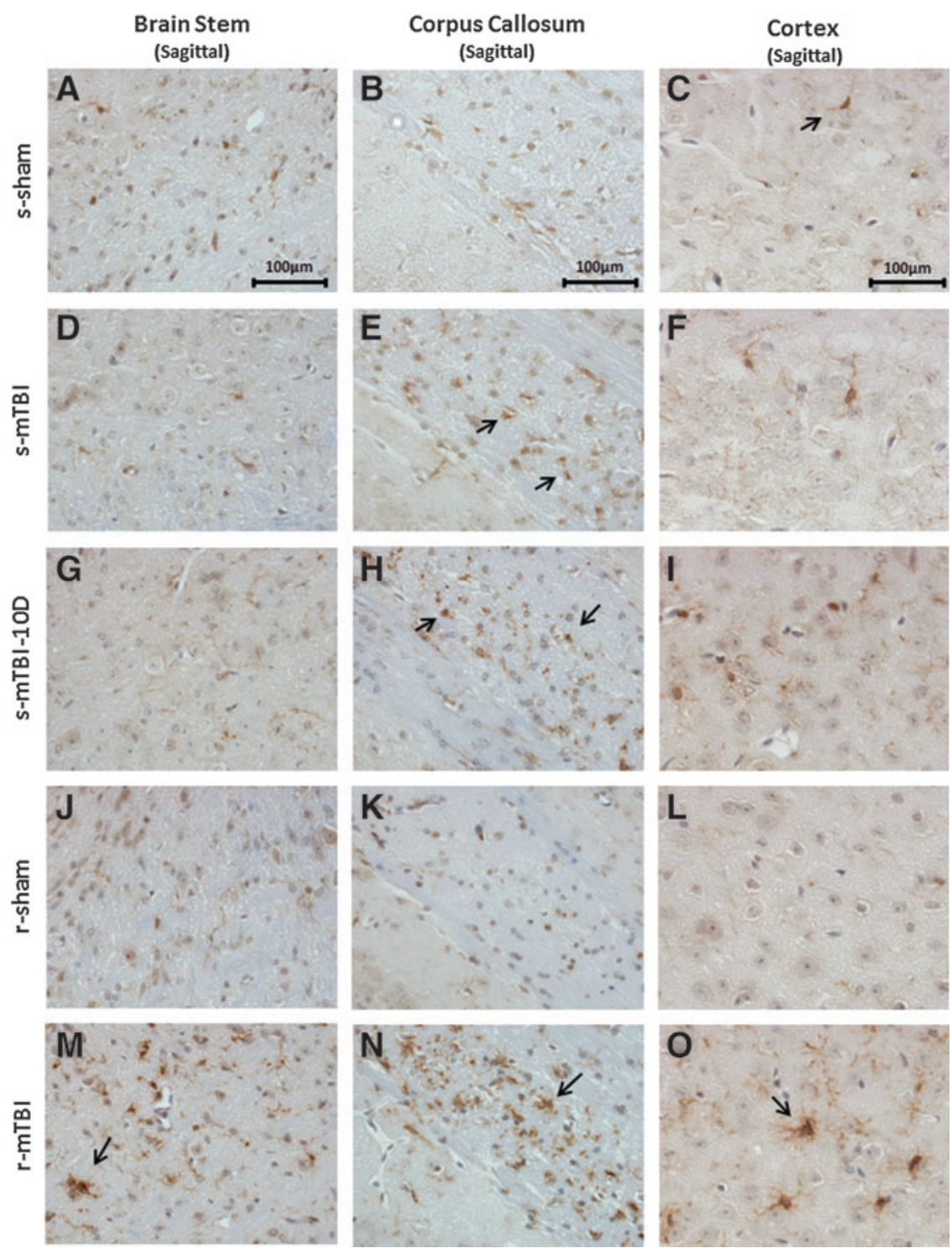

FIG. 11. Immunohistochemical labeling for microglia with anti-Iba1. Sagittal sections of the mouse brain at $\sim 0.4 \mathrm{~mm}$ lateral to midline in the brainstem (A, D, G, J, M), in the corpus callosum $(\mathbf{B}, \mathbf{E}, \mathbf{H}, \mathbf{K}, \mathbf{N})$, and in the cortex $(\mathbf{C}, \mathbf{F}, \mathbf{I}, \mathbf{L}, \mathbf{O})$. There was no microglial activation in the sham $(\mathbf{A}-\mathbf{C})$ and r-sham $(\mathbf{J}-\mathbf{L})$ groups. An increased area of anti-Iba1 immunoreactivity was observed in the corpus callosum at $24 \mathrm{~h}(\mathbf{E})$ and 10 days $(\mathbf{H})$ post s-mTBI. Anti-Iba1 immunohistochemistry indicates multifocal microglial activation after r-mTBI in the brainstem $(\mathbf{M})$, corpus callosum $(\mathbf{N})$, and cortex $(\mathbf{O})$. Tissue sections were counterstained with hematoxylin. Color image is available online at www.liebertonline.com/neu
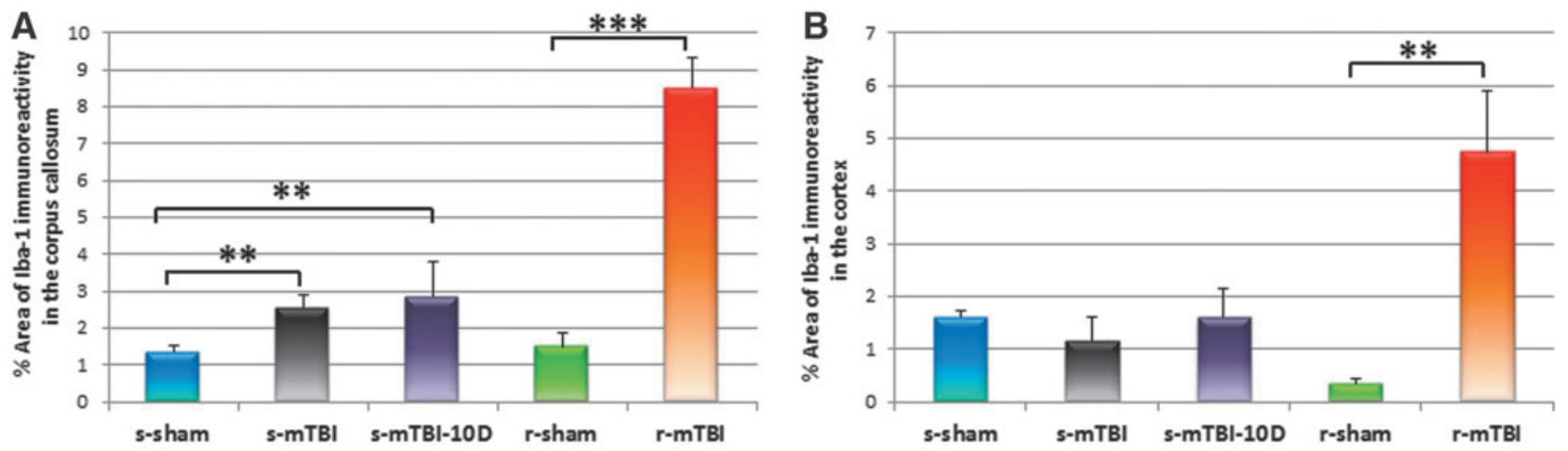

FIG. 12. Quantitative analysis of anti-Iba1 staining intensity in three $100 \mu \mathrm{m}^{2}$ areas of the corpus callosum (A) and two $200 \mu \mathrm{m}^{2}$ areas of the cortex (B) $(n=4)$. Data are presented as mean \pm SEM, $(* * p<0.001$; *** $p<0.0001)$. Color image is available online at www.liebertonline.com/neu 
Table 1. Summary of the Location and Relative INTENSITY OF IMMUNOHISTOCHEMICAL DATA FOR AMYLOID Precursor Protein (A), Glial Fibrillary Acid Protein (G), And Anti-Iba1 (M)

\begin{tabular}{llllll}
\hline & $s$-sham & $s-m$ TBI & $s-m$ TBI-10D & r-sham & r-mTBI \\
\hline \multirow{2}{*}{ Cortex } & - & - & - & - & A \\
& - & - & - & G & G G G \\
\multirow{5}{*}{ Corpus callosum } & - & - & - & - & M M \\
& - & A A A & - & - & AA \\
\multirow{5}{*}{ CA1 } & - & - & G G & G & G G G \\
& M & M M & M M & M & M M M \\
\multirow{3}{*}{ Brainstem } & - & - & - & - & - \\
& G & G & G & G G & G G G \\
& M & M & M & M & M \\
& - & A & - & - & A A \\
& - & - & - & - & - \\
\hline
\end{tabular}

Present $(\mathrm{X})$, moderate staining (XX), and intense staining (XXX). s-mTBI, single mild traumatic brain injury; r-mTBI, repetitive mild traumatic brain injury.

morphological assessment with $\mathrm{H} \& \mathrm{E}$ and $\mathrm{LFB} / \mathrm{CV}$ staining, including no signs of neuronal loss. Axonal injury following trauma results in swollen and tortuous axons that can be detected by the accumulation of APP as early as 35 min after brain injury, ${ }^{46}$ and in our model, a single impact resulted in APP-immunoreactive axons localized to the $\mathrm{CC}$, evident at $24 \mathrm{~h}$ after injury. By 10 days postinjury (s-mTBI-10D) there was no evidence for APP immunoreactivity, consistent with the current literature for animal models in that strong APP staining is present at $24 \mathrm{~h}$ post-injury, ${ }^{22,30,47}$ which diminishes by 14 days post-injury. ${ }^{42,46}$ In the r-mTBI group, more widespread damage was observed, with immunoreactive axons also evident in the spinal trigeminal tracts of the BS and beneath the impact site in the form of APP immunoreactive neuronal perikaryas. However, in the r-mTBI group, the APP immunoreactive axonal swellings were less frequent and less robustly stained in the $\mathrm{CC}$ than those observed at $24 \mathrm{~h}$ after s-mTBI. These observations may be consistent with a deleterious effect of repetitive versus single injury on the ability of the brain to efficiently catabolize and clear APP, facilitating amyloidogenic processing and contributing to later evolution of amyloid plaque pathology. ${ }^{17,48}$ More work is required to explain why no increase in APP immunoreactivity was observed in r-mTBI versus s-mTBI. Nevertheless, the comparison of r-mTBI with s-mTBI-10D (where both groups are matched for time since the first/single injury) clearly demonstrates that repetitive injury sustains an APP immunoreactive axonal profile over the 10-day period following the initial injury.

In addition to the white matter damage, the r-mTBI group was associated with a notable reactive astrocytosis, with a distinct pattern of GFAP-positive reactive astrocytes localized to the medium and deep layers of the cortex beneath the impact site. As observed in the s-mTBI-10D group, our model produced a measurable reactive astrocytosis at 10 days after initial mTBI. These results are consistent with other studies that showed astrocytosis peaking between 10 and 14 days following concussion in rodents. ${ }^{42,49,50}$ This increase in gliosis is also believed to coincide with macrophage accumulation, which has also been shown to peak at 10 days post- injury. Our data demonstrate that repetitive, noninvasive $\mathrm{mTBI}$ in the mouse resulted in a graded injury response, with the extent of reactive astrocytosis increased in animals exposed to repetitive impacts. Whereas a single impact produced moderate reactive astrocytosis at 10 days post-injury and was isolated to the $\mathrm{CC}$, five impacts over 9 days showed increased staining in the $\mathrm{CC}$ as well as cortical and hippocampal involvement.

As well as axonal injury and astrogliosis, microgliosis was also detected in the CC of injured animals as early as $24 \mathrm{~h}$ and up to at least 10 days post-mTBI, whereas the r-mTBI group revealed clusters of activated microglia in multiple brain regions (CC, BS, and cortex). The time course of microglial activation observed in this study was similar to that noted in previous reports. ${ }^{31,51-53}$ Depending mainly upon injury severity and the duration of the ensuing inflammatory cascade, microgliosis can be either beneficial or detrimental with respect to tissue preservation. ${ }^{54}$ Known as the first line of defense in both focal and diffuse TBI ${ }^{55}$ microglial activation has been reported to play a beneficial role by secreting brain-derived neurotrophic growth factors and insulin-like growth factor. ${ }^{56-58}$ They have also been shown to have a detrimental role at an acute time point by secreting inflammatory cytokines such as interleukin (IL)- $1 \beta$ and tumor necrosis factor (TNF)- $\alpha .^{59,60}$ Moreover, in several TBI models, minocycline, which has antiinflammatory properties, has been shown to reduce histopathological consequences after mTBI in mice ${ }^{61,62}$ Of particular interest is a recent study by Siopi and collaborators that demonstrated that reducing neuroinflammation post-injury attenuated memory impairment in a mouse model of $\mathrm{CHI} ;{ }^{63,64}$ however, more work is required to address the impact of microglial activation on cognitive function after TBI.

\section{Conclusion}

In conclusion, we have developed a simple and reproducible mouse model of mTBI, which induces pathological and behavioral features comparable to those observed in the human condition. Moreover, by recapitulating the pathological alterations observed in human TBI patients, this model is also suitable for studying various mechanisms of post-traumatic injury such as axonal injury, cell death, or apoptosis. Moving forward, this model of mTBI can be used to investigate the long term consequences of r-mTBI, the influence of different numbers of injuries and different inter-injury intervals, and the impact of r-mTBI in mice genetically modified at loci of relevance to human TBI such as Tau or APOE, aspects currently lacking in the literature. An increased understanding of mTBI and its cumulative effects will enable identification of molecular targets specific to mTBI and ultimately the development of novel, effective therapeutics, which are desperately needed.

\section{Acknowledgments}

This research was funded by a Department of Defense award (W81XWH-10-1-0759) to Dr. Fiona Crawford and by the Roskamp Foundation.

\section{Author Disclosure Statement}

No competing financial interests exist.

\section{References}

1. Faul, M., Xu, L., Wald, M.M., and Coronado, V.G. (2010). Traumatic Brain Injury in The United States: Emergency Department Visits, Hospitalizations, and Deaths 2002-2006. Centers for Disease Control and Prevention, National Center for Injury Prevention and Control: Atlanta.

2. Corsellis J.A., Bruton, C.J., Freeman-Browne, D. (1973). The aftermath of boxing. Psychol. Med. 3, 270-303.

3. Guskiewicz, K.M., McCrea, M., Marshall, S.W., Cantu, R.C., Randolph, C., Barr, W., Onate, J.A., and Kelly, J.P. (2003). Cumulative 
effects associated with recurrent concussion in collegiate football players: the NCAA concussion study. JAMA 290, 2549-2555.

4. Guskiewicz, K.M., Marshall, S.W., Bailes, J., McCrea, M., Cantu, R.C., Randolph, C., and Jordan, B.D. (2005). Association between recurrent concussion and late-life cognitive impairment in retired professional football players. Neurosurgery 57, 719-726.

5. Guskiewicz, K.M., Marshall, S.W., Bailes, J., McCrea, M., Harding, H.P., Matthews, A., Mihalik, J.R., and Cantu, R.C. (2007). Recurrent concussion and risk of depression in retired professional football players. Med. Sci. Sports Exerc. 39, 903-909.

6. Omalu, B.I., DeKosky, S.T., and Minster, R.L. (2005). Chronic traumatic encephalopathy in a national football league player. Neurosurgery $57,128-134$.

7. Omalu, B.I, DeKosky, S.T., and Hamilton, R.L. (2006). Chronic traumatic encephalopathy in a national football league player: part II. Neurosurgery 59, 1086-1092.

8. Omalu, B.I., Hamilton, R.L., Kamboh, M.I., DeKosky, S.T., and Bailes J. (2010). Chronic traumatic encephalopathy in a national football league player: case report and emerging medico-legal practice questions. J. Forensic Nurs. 6, 40-46.

9. Omalu, B.I., Fitzsimmons, R.P., Hammers, J., and Bailes, J. (2010) Chronic traumatic encephalopathy in a professional American wrestler. J. Forensic Nurs. 6, 130-136.

10. Omalu, B., Bailes, J., Hamilton, R.L., Kamboh, M.I., Hammers, J., Case, M., and Fitzsimmons, R. (2011). Emerging histomorphologic phenotypes of chronic traumatic encephalopathy in American athletes. Neurosurgery $69,173-183$

11. Broglio, S.P., Eckner, J.T., Martini, D., Sosnoff, J.J., Kutcher J.S., and Randolph, C. (2011). Cumulative head impact burden in high school football. J. Neurotrauma 28, 2069-2078.

12. Gavett, B.E., Stern, R.A., Cantu, R.C., Nowinski, C.J., and McKee, A.C. (2010). Mild traumatic brain injury: a risk factor for neurodegeneration. Alzheimers Res. Ther. 2, 18

13. Gavett, B.E., Stern, R.A., and McKee, A.C. (2011). Chronic traumatic encephalopathy: a potential late effect of sport-related concussive and subconcussive head trauma. Clin. Sports Med. 30, 179-188.

14. Stern, R.A., Riley, D.O., Daneshvar, D.H., Nowinski, C.J., Cantu, R.C., and McKee, A.C. (2011). Long-term consequences of repetitive brain trauma: chronic traumatic encephalopathy. PM R 3, Suppl. 2, S460-467.

15. McKee, A.C., Cantu, R.C., Nowinski, C.J., Hedley-Whyte, E.T., Gavett, B.E., Budson, A.E., Santini,V.E., Lee, H.S., Kubilus, C.A., and Stern, R.A. (2009). Chronic traumatic encephalopathy in athletes: progressive tauopathy after repetitive head injury. J. Neuropathol. Exp. Neurol. 68, 709-735.

16. Costanza, A., Weber, K., Gandy, S., Bouras, C., Hof, P.R., Giannakopoulos, P., and Canuto, A. (2011). Contact sport-related chronic traumatic encephalopathy in the elderly: clinical expression and structural substrates. Neuropathol. Appl. Neurobiol. 37, 570-584.

17. Johnson, V.E., Stewart, W., and Smith, D.H. (2010). Traumatic brain injury and amyloid- $\beta$ pathology: a link to Alzheimer's disease? Nat. Rev. Neurosci. 11, 361-370.

18. Van Den Heuvel, C., Thornton, E., and Vink, R. (2007). Traumatic brain injury and Alzheimer's disease: a review. Prog. Brain Res, 161, 303-316.

19. Förstl, H., Haass, C., Hemmer, B., Meyer, B., and Halle, M. (2010). Boxing-acute complications and late sequelae: from concussion to dementia. J. Forensic Nurs. 6, 40-46.

20. Raghupathi, R., and Margulies, S.S. (2002). Traumatic axonal injury after closed head injury in the neonatal pig. J. Neurotrauma 19, 843-853.

21. Raghupathi, R., Mehr, M., Helfaer, M.A., and Margulies, S.S. (2004). Traumatic axonal injury is exacerbated following repetitive closed head injury in the neonatal pig. J. Neurotrauma 21, 307-316.

22. Browne, K.D., Chen, X.H., Meaney, D.F., and Smith, D.H. (2011) Mild traumatic brain injury and diffuse axonal injury in swine. J. Neurotrauma 28, 1747-1755.

23. Gennarelli, T.A., Thibault, L.E., Adams, J.H., Graham, D.I., Thompson, C.J., and Marcincin, R.P. (1982). Diffuse axonal injury and traumatic coma in the primate. Ann. Neurol. 12, 564-574.

24. Laurer, H.L., Bareyre, F.M., and Lee, V.M. (2001). Mild head injury increasing the brain's vulnerability to a second concussive impact. J. Neurosurg. 95, 859-870

25. DeFord, S.M., Wilson, M.S., and Rice, A.C. (2002). Repeated mild brain injuries result in cognitive impairment in $\mathrm{B} 6 \mathrm{C} 3 \mathrm{~F} 1$ mice. J. Neurotrauma 19, 427-438
26. Uryu, K., Laurer, H., McIntosh, T., Praticò, D., Martinez, D., Leight, S., Lee, V.M., and Trojanowski, J.Q. (2002). Repetitive mild brain trauma accelerates Abeta deposition, lipid peroxidation, and cognitive impairment in a transgenic mouse model of Alzheimer amyloidosis. J. Neurosci. 22, 446-454.

27. Longhi, L., Saatman, K.E., Fujimoto, S., Raghupathi, R., Meaney, D.F., Davis, J., McMillan, B.S., Conte, V., Laurer, H.L., Stein, S., Stocchetti, N., and McIntosh, T.K. (2005).Temporal window of vulnerability to repetitive experimental concussive brain injury. Neurosurgery $56,364-374$

28. Creeley, C.E., Wozniak, D.F., Bayly, P.V., Olney, J.W., and Lewis, L.M. (2004). Multiple episodes of mild traumatic brain injury result in impaired cognitive performance in mice. Acad. Emerg. Med. 11, 809819.

29. Nakajima, Y., Horiuchi, Y., Kamata, H., Yukawa, M., Kuwabara, M., and Tsubokawa, T. (2010). Distinct time courses of secondary brain damage in the hippocampus following brain concussion and contusion in rats. Tohoku J. Exp. Med. 221, 229-235.

30. Prins, M.L., Hales, A., Reger, M., Giza, C.C., and Hovda, D.A. (2010). Repeat traumatic brain injury in the juvenile rat is associated with increased axonal injury and cognitive impairments. Dev. Neurosci. 32, 510-518.

31. Shitaka, Y., Tran, H.T., Bennett, R.E., Sanchez, L., Levy, M.A., Dikranian, K., and Brody, D.L. (2011). Repetitive closed-skull traumatic brain injury in mice causes persistent multifocal axonal injury and microglial reactivity. J. Neuropathol. Exp. Neurol. 70, $551-567$

32. Cole, J.T., Yarnell, A., Kean, W.S., Gold, E., Lewis, B., Ren, M., McMullen, D.C., Jacobowitz, D.M., Pollard, H.B., O'Neill, J.T., Grunberg, N.E., Dalgard, C.L., Frank, J.A., and Watson, W.D. (2011) Craniotomy: true sham for traumatic brain injury, or a sham of a sham? J. Neurotrauma 28, 359-369.

33. Brody, D.L., Mac Donald, C., Kessens, C.C., Yuede, C., Parsadanian, M., Spinner, M., Kim, E., Schwetye, K.E., Holtzman, D.M., and Bayly, P.V. (2007). Electromagnetic controlled cortical impact device for precise, graded experimental traumatic brain injury. J. Neurotrauma $24,657-673$

34. Albert-Weissenberger, C., and Siren, A.L. (2010). Experimental traumatic brain injury. Exp. Transl. Stroke Med. 2, 16.

35. Conte, V., Uryu, K., Fujimoto, S., Yao, Y., Rokach, J., Longhi, L., Trojanowski, J. Q., Lee, V. M.-Y., McIntosh, T. K., and Praticò, D. (2004). Vitamin E reduces amyloidosis and improves cognitive function in $\mathrm{Tg} 2576$ mice following repetitive concussive brain injury. J. Neurochem. 90, 758-764.

36. Yoshiyama, Y., Uryu, K., Higuchi, M., Longhi, L., Hoover, R., Fujimoto, S., McIntosh, T., Lee, V.M., and Trojanowski, J.Q. (2005). Enhanced neurofibrillary tangle formation, cerebral atrophy, and cognitive deficits induced by repetitive mild brain injury in a transgenic tauopathy mouse model. J. Neurotrauma 22, 1134-1141

37. Kane, M.J., Angoa-Pérez, M., Briggs, D.I., Viano, D.C., Kreipke, C.W., and Kuhn, D.M. (2012). A mouse model of human repetitive mild traumatic brain injury. J. Neurosci. Methods 203, 41-49.

38. Franklin, K.B.J., and Paxinos, G. (2001). The Mouse Brain in Stereotaxic Coordinates, 2 nd ed. Elsevier/Academic Press: San Diego, London.

39. Ruifrok, A.C., and Johnston, D.A. (2001). Quantification of histochemical staining by color deconvolution. Anal. Quant. Cytol. Histol. 23, 291-299.

40. Park, H.K., Fernandez, I.I., Dujovny, M., and Diaz, F.G. (1999). Experimental animal models of traumatic brain injury: medical and biomechanical mechanism. Crit. Rev. Neurosurg. 9, 44-52.

41. LaPlaca, M.C., Simon, C.M., Prado, G.R., and Cullen, D.K. (2007). CNS injury biomechanics and experimental models. Prog. Brain Res. $161,13-26$.

42. Huh, J.W., Widing, A.G., and Raghupathi, R. (2008).Midline brain injury in the immature rat induces sustained cognitive deficits, bihemispheric axonal injury and neurodegeneration. Exp. Neurol. 213, 8492.

43. Guskiewicz, K.M. (2011). Balance assessment in the management of sport-related concussion. Clin. Sports Med. 30, 89-102.

44. Shultz, S.R., Bao, F., Omana, V., Chiu, C., Brown, A., and Cain, D.P (2012). Repeated mild lateral fluid percussion brain injury in the rat causes cumulative long-term behavioral impairments, neuroinflammation, and cortical loss in an animal model of repeated concussion. J. Neurotrauma 29, 281-294. 
45. Eakin, K., and Miller, J.P. (2012). Mild traumatic brain injury is associated with impaired hippocampal spatiotemporal representation in the absence of histological changes. J. Neurotrauma 29, 1180-1187.

46. Pierce, J.E., Trojanowski, J.Q., Graham, D.I., Smith, D.H., and McIntosh, T.K. (1996). Immunohistochemical characterization of alterations in the distribution of amyloid precursor proteins and betaamyloid peptide after experimental brain injury in the rat. J. Neurosci. 16, 1083-1090.

47. Huh, J.W., Widing, A.G., and Raghupathi, R. (2007). Basic science; repetitive mild non-contusive brain trauma in immature rats exacerbates traumatic axonal injury and axonal calpain activation: a preliminary report. J. Neurotrauma 24, 15-27.

48. Sivanandam, T.M., and Thakur, M.K. (2012). Traumatic brain injury: a risk factor for Alzheimer's disease. Neurosci. Biobehav. Rev. 36 , 1376-1381.

49. Hamberger, A., Viano, D.C., Säljö, A., and Bolouri, H. (2009). Concussion in professional football: morphology of brain injuries in the NFL concussion model-part 16. Neurosurgery 64, 1174-1182.

50. Hellewell, S.C., Yan, E.B., Agyapomaa, D.A., Bye, N., and MorgantiKossmann, M.C. (2010). Post-traumatic hypoxia exacerbates brain tissue damage: analysis of axonal injury and glial responses. J. Neurotrauma 27, 1997-2010.

51. Kelley, B.J., Lifshitz, J., and Povlishock, J.T. (2007). Neuroinflammatory responses after experimental diffuse traumatic brain injury. J. Neuropathol. Exp. Neurol. 66, 989-1001.

52. Chen, S., Pickard, J.D., and Harris, N.G. (2003). Time course of cellular pathology after controlled cortical impact injury. Exp. Neurol. 182, 87-102.

53. Haselkorn, M.L., Shellington, D.K., Jackson, E.K., Vagni, V.A., Janesko-Feldman, K., Dubey, R.K., Gillespie, D.G., Cheng, D., Bell, M.J., Jenkins, L.W., Homanics, G.E., Schnermann, J., and Kochanek, P.M. (2010). Adenosine A1 receptor activation as a brake on the microglial response after experimental traumatic brain injury in mice. J. Neurotrauma 27, 901-910.

54. Neumann, H., Kotter, M.R., and Franklin, R.J. (2009). Debris clearance by microglia: an essential link between degeneration and regeneration. Brain. 132 (Pt. 2), 288-295.

55. Morganti-Kossmann, M.C., Rancan, M., Stahel, P.F., and Kossmann, T. (2002). Inflammatory response in acute traumatic brain injury: a double-edged sword. Curr. Opin. Crit. Care 8, 101-105.

56. Neumann, J., Gunzer, M., Gutzeit, H.O., Ullrich, O., Reymann, K.G., and Dinkel, K. (2006). Microglia provide neuroprotection after ischemia. FASEB J. 20, 714-716.
57. Lalancette-Hebert, M., Gowing, G., Simard, A., Weng, Y.C., and Kriz, J. (2007). Selective ablation of proliferating microglial cells exacerbates ischemic injury in the brain. J. Neurosci. 27, 2596-2605.

58. Thored, P., Heldmann, U., Gomes-Leal, W., Gisler, R., Darsalia, V., Taneera, J., Nygren, J.M., Jacobsen, S.E.W., Ekdahl, C.T., Kokaia, Z., and Lindvall, O. (2009). Long-term accumulation of microglia with proneurogenic phenotype concomitant with persistent neurogenesis in adult subventricular zone after stroke. Glia 57, 835-849.

59. Lloyd, E., Somera-Molina, K., Van Eldik, L.J., Watterson, D.M., and Wainwright, M.S. (2008). Suppression of acute proinflammatory cytokine and chemokine upregulation by post-injury administration of a novel small molecule improves long-term neurologic outcome in a mouse model of traumatic brain injury. J. Neuroinflammation 30, 28.

60. Schmidt, O., Heyde, C., Ertel, W., and Stahel, P. (2005). Closed head injury-an inflammatory disease? Brain Res. Rev. 48, 388-399.

61. Homsi, S., Piaggio, T., Croci, N., Noble, F., Plotkine, M., MarchandLeroux, C., and Jafarian-Tehrani, M. (2010). Blockade of acute microglial activation by minocycline promotes neuroprotection and reduces locomotor hyperactivity after closed head injury in mice: a twelve-week follow-up study. J. Neurotrauma 27, 911-921.

62. Siopi, E., Cho, A.H., Homsi, S., Croci, N., Plotkine, M., MarchandLeroux, C., and Jafarian-Tehrani, M. (2011). Minocycline restores $\operatorname{sAPP} \alpha$ levels and reduces the late histopathological consequences of traumatic brain injury in mice. J. Neurotrauma 28, 2135-2143.

63. Siopi, E., Calabria, S., Plotkine, M., Marchand-Leroux, C., and Jafarian-Tehrani, M. (2012). Minocycline restores olfactory bulb volume and olfactory behavior after traumatic brain injury in mice. J. Neurotrauma 29, 354-361.

64. Siopi, E., Llufriu-Dabén, G., Fanucchi, F., Plotkine, M., MarchandLeroux, C., and Jafarian-Tehrani, M. (2012). Evaluation of late cognitive impairment and anxiety states following traumatic brain injury in mice: the effect of minocycline. Neurosci. Lett. 511, 110-115.

Address correspondence to: Benoit Mouzon, M.S.

Roskamp Institute 2040 Whitfield Avenue Sarasota, FL 34243

E-mail: bmouzon@rfdn.org 